\title{
Internet-Based Robot Assisted RF and Wireless Laboratory for Engineering Education
}

\author{
http://dx.doi.org/10.3991/ijoe.v8i3.2023 \\ Ananda Maiti ${ }^{1}$ and Subhasis Mahata ${ }^{2}$ \\ ${ }^{1}$ Vellore Institute of Technology, Vellore, India \\ ${ }^{2}$ Indian Institute of Technology, Kharagpur, India
}

\begin{abstract}
The rapid growth of modern Radio Frequency (RF) and wireless communication systems requires experienced professionals in the RF and wireless areas. In the curriculum of engineering education, many university departments are introducing courses on high frequency telecommunication and/or radio communication related courses to equip students to meet the needs of industry. Effective learning can only be achieved by combining theoretical course with a corresponding laboratory. Development of an Internet-based RF communication laboratory is reported. Some experiments in a RF and wireless communication laboratory often require change in experimental setup, which is accomplished in a conventional laboratory by the technical personnel. However, in a remote triggered web-based laboratory, this is not possible, and change in experimental setup can only be realized by using an Intelligent robot. In this paper, we report on the design and development of a robot-assisted remote-controlled modern RF and wireless communication laboratory available online $(24 \times 7)$ over the internet.
\end{abstract}

Index Terms-Remote laboratory, radio frequency, wireless, robot control, web-services, LabVIEW

\section{INTRODUCTION}

Currently wireless communications technology is one of the most rapidly growing disciplines in electrical engineering. The telecommunication industry is also shifting away from wired networks to an era of wireless technology. RF and wireless technology has been recognized as an essential core subject of EE education [1]. It is thus important to prepare both the undergraduate and postgraduate students for careers in wireless communication technology to meet the needs of the telecommunications industry. One of the objectives of the $\mathrm{RF}$ and wireless laboratory is to provide the students with hands-on laboratory experience which may include:

- Basic RF concepts

- RF circuit design concept

- RF communication system concept

- Building of RF circuits

- Characterization of RF circuits

- Usage of RF measuring instruments

- Measurement automation

It is important to develop wireless and RF laboratory that will provide the opportunity to acquire both in-depth theoretical understanding and hands-on laboratory experience. A review of the published literature over the past decade reveals that many University departments offer laboratory courses relating to $\mathrm{RF}$ and wireless communications [2]. As the equipment required in high frequency telecom/radio frequency laboratories is very expensive and delicate, most educational institutions cannot afford to have such equipment and trained personnel to maintain them. As a relatively low cost solution, the RF Courseware modules are being made available. For example, Dream Catcher offers ME1000 RF Circuit Design [3], which enables training in the areas of RF and wireless communication. In this paper, a framework for the development and implementation of an intelligent robot assisted Java-based remote controlled RF and wireless laboratory, accessible via the Internet by means of a Web browser is described. The paper is organized in five sections: Section II introduces the problems and limitations of conventional RF and wireless laboratory training and describes remote laboratory options to overcome these problems and/or limitations. Section III describes the implementation of the RF laboratory. Description of the robotics module is covered in Section IV. A case study is presented in Section V followed by the conclusions in Section VI.

\section{RF AND WIRELESS LABORATORY}

The major limitations for the development of a RF laboratory are the high setup and maintenance costs of the $\mathrm{RF}$ and microwave equipments and trained workforce for supporting them. Commonly used RF measurement instruments include vector signal analyzer (VSA), RF signal generators (SG), network analyzers (NA) and spectrum analyzers (SA) which are very expensive. Remote triggered laboratories are becoming a potential alternative to classical laboratories, which provide similar learning experience [4-7]. Internet and especially the World Wide Web provide a ubiquitous medium for connecting devices and robots for remote operation. Internet is being used by many universities to cope with the common problem faced by institutions concerning the limited availability of expensive laboratory equipment.

It should be noted that in some experiments of the RF laboratory, it is often necessary to change connections and/or modify the setup, which is easy in conventional laboratory as these are done by laboratory technicians. In a remote triggered RF laboratory, this is not possible. However, change in experimental setup can be made by an intelligent robot. For example, robots could be programmed to perform tasks, such as, move forward and/or reverse (for example, distance variation in antenna experiments) to study antenna gain as a function of distance between the source and the receiver. The scope of 
a remote RF laboratory can thus be extended by using programmable robots. Deploying RF experiments in remote laboratories, based on the web and Internet technologies is thus a cost effective and relatively safe approach to provide RF laboratory facility. These laboratories can be made available $(24 \times 7)$ to geographically distributed students in different locations for remote access and perform experiments.

The requirements for implementing a remote $\mathrm{RF}$ laboratory have been studied previously [8-11]. While implementing an online educational tool, it must be remembered that the target is mostly unskilled or novice users. The development of robot-assisted laboratories remotely accessible through the Internet is a rather complex task as it involves expertise from multiple disciplines such as remote operation of robotics, intelligent control, human-computer interaction and webbased intelligent programming. For a robot-assisted laboratory, it is necessary to gain experience about the control of the robot involved in the experimental setup and at the same time to perform the experiment itself.

\section{LABORATORY SETUP}

The Laboratory consists of several components (see Figure 1) that combine together to initiate, operate and acquire data from the experiments. These components are:

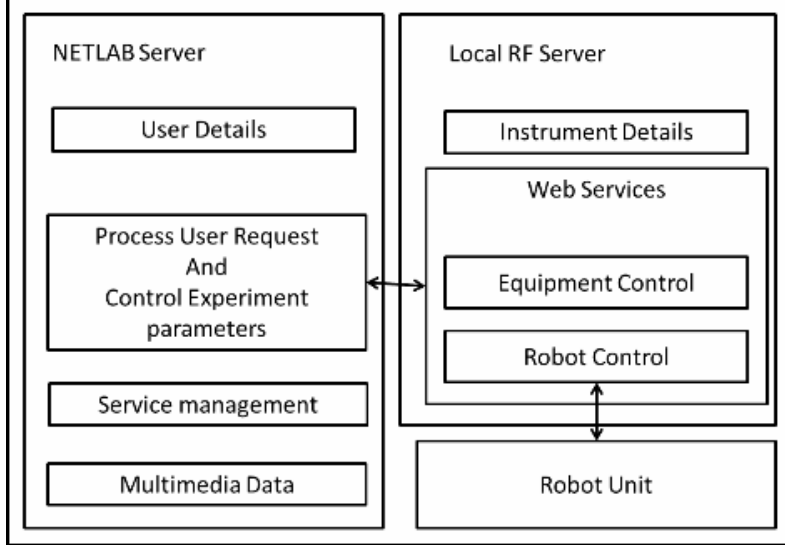

Figure 1. The laboratory setup (flow).

\section{A. NETLab Server}

The NETLab Server is responsible for the following:

a) Maintaining the user details.

b) Accepting and processing User Request.

c) Scheduling: Since multiple users cannot use the instrument simultaneously, there are scheduling schemes to proper handle user requests. For the RF experiments, a simple Time-Slot method is applied, where only one user can book a time slot and is allowed to control the experiment in that particular time slot. This is because these experiments are interactive and requires constant vigilance from the

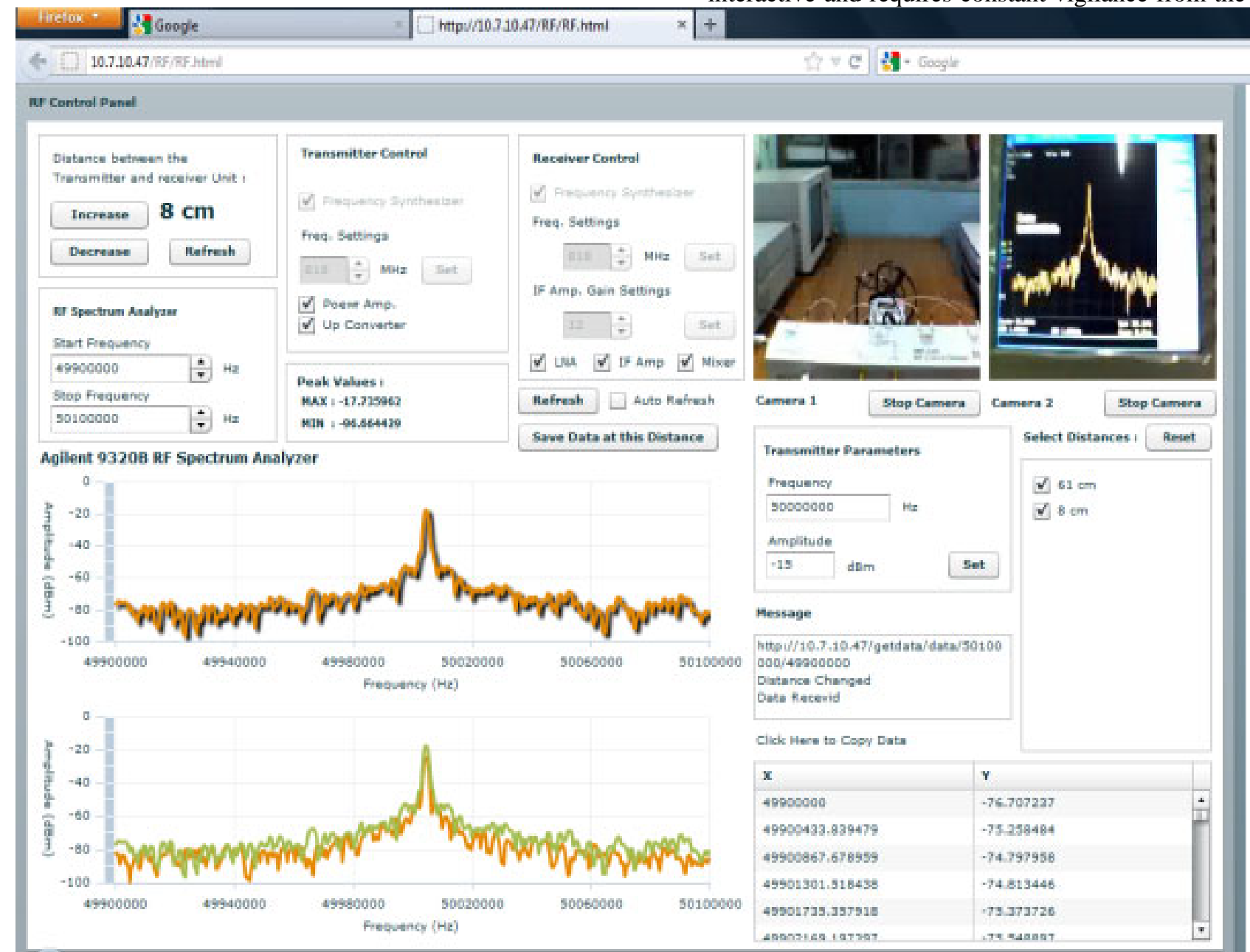

Figure 2. The user-client page of the remote laboratory. 
user's side.

d) Service Management: The NETLab Server connects to the Local RF server through a set of web services. There is a set of web services to control the entire setup:

- To measure data from the analyzer and control the RF output equipment.

- To control the different functions of the Device under test (DUT).

- To control the power control unit.

- To gather data after an experiment request is successfully processed.

- To control the robotic unit.

e) Multimedia Accessories: In the current version of the laboratory management system (LaMS) the functions included are: Manuals (pre-laboratory and experiment) contain detailed information about the experiment; the video/image section contains videos and images about different aspects of the experiment; equipment detail section contains the information about the instruments used in a particular experiment; interactive animation section includes animations that would simulate the setting up of the experiment; sections involving question box and video chatting provide communication with the instructor and also the other students; each experiment contains an evaluation procedure page [12] such as, viva voce and laboratory report submission.

f) The user interface: Users interact with Web-based thin-client graphical user interfaces (GUI) to control the test equipment and check/download the data/results. The (GUI) is developed in Adobe FLEX. The interface is hosted in the server and provides a complete control for the operation of the instruments through web services (see Fig. 2).

\section{B. Local RF Server}

The local server is located at the site of the RF instruments, and is responsible for actual control of the experimental parameters. The Local server receives requests from the NETLab server as web services, runs the instruments such as, the signal generator and spectrum analyzer and returns the data in XML format. Different $\mathrm{RF}$ experiments require different types of components and equipment connections. In some experiments, RF switches may be used which however, is very expensive. In such cases robots may be used to provide peripheral control of the equipment to the user. The local RF Server's job is to control the robotics as well.

\section{Robotic Control Unit}

The robot control unit (RCU) is different; from one experiment to another. For example, one experiment may require linear motion of transmitters and receivers while other experiment may require angular motion for rotating the antenna. The RCU needs to be designed accordingly.

\section{Instrument Power Control Unit}

As the equipments are very expensive, they should be powered only during the experiment. They are only turned 'on' when the user is ready to perform the experiment. This is done using a power control circuit (PCU) which is connected to a power control server in the NETLab system. The NETLab server powers the instruments 'on' through Web Services when the user's time slot is within 5 minutes.

\section{E. RF Equipment}

The RF Measurement Equipment used in the laboratory is Agilent N9310B RF signal generator and N9320B RF spectrum analyzer. A transmit/receive unit from Dream Catcher is also used. All these equipments are controlled by LabVIEW from National Instruments.

\section{RoBOTIC Module}

Robots are employed to perform 'similar to human' actions such as, pick up objects and moving the equipment. However, the main problem with internetbased remote operation is the unpredicted delay unlike in traditional remote operation where interfaces have fixed delays. It is thus necessary to develop interfaces employing advanced web technologies and web development platforms such as LabVIEW [13, 14].

For change and modification in the experimental setup, LEGO MINDSTORM NXT robots are used. LEGO MINDSTORM NXT consists of a set of tools and robotic parts that may be assembled for various operations. As an example, a trolley to carry equipment/components to change the distance between the receiver and the

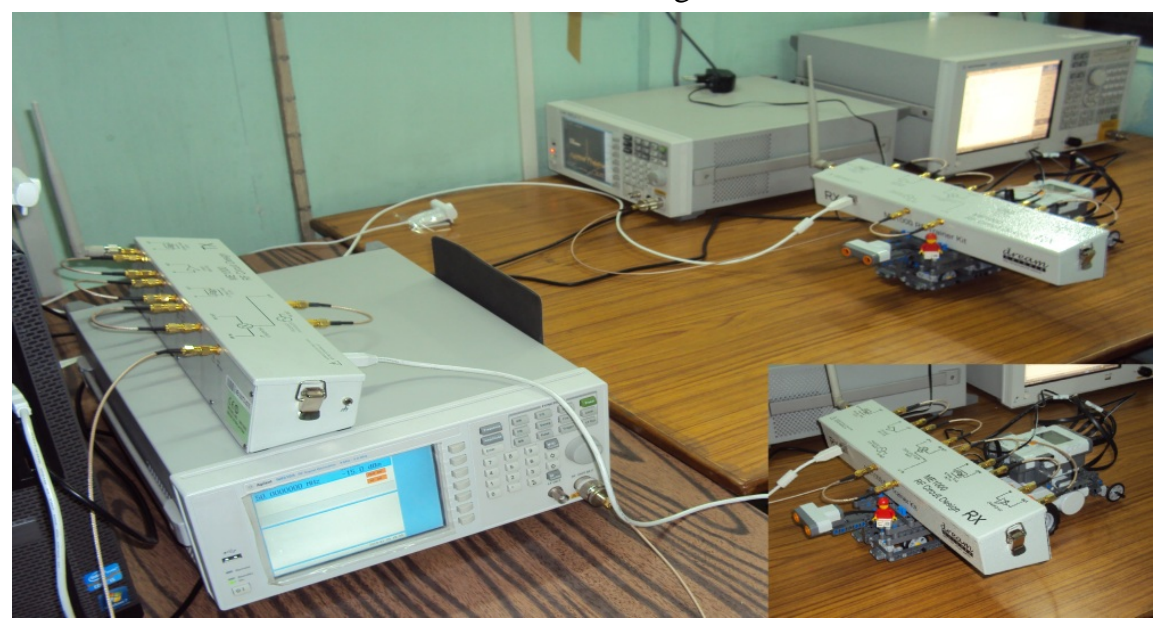

Figure 3. Typical laboratory setup for an experiment. (Inset) The robot control unit. 
transmitter. The detail of NXT based robot specifications is shown in Table 1. LabVIEW 2009 provides a module for NXT, which is used to control the servos and sensors connected to the NXT.

TABLE I.

SUMMARY OF NXT BASED ROBOT SPECIFICATIONS.

\begin{tabular}{|c|c|}
\hline Max. Handling Capacity & $14 \mathrm{~kg}$ \\
\hline Robot Weight & $1 \mathrm{~kg}$ \\
\hline Max. Reach & $1 \mathrm{~m}$ \\
\hline Max. Speed & $1 \mathrm{~cm} / \mathrm{s}$ \\
\hline Finest Movement Control & $1 \mathrm{~cm}$ \\
\hline Degrees of Freedom & 1 \\
\hline
\end{tabular}

The maximum handling capacity is $14 \mathrm{~kg}$ refers to the maximum weight the robot can pull or push. The robot has a truck as trailer framework to mount the transmitter or receiver. Degrees of freedom is 1 ; which means that this is the maximum number of variables that is available to bring the robot in a new position or the minimum number of variables required to define the position of the robot relative to its origin. In this case, the degree of freedom is 1 , since the robot is required to move only forward or backward for the antenna gain experiment. The NXT robot is named with a 'unique string' that LabVIEW recognizes for controlling the robot. Several sub-VIs are made available in the NXT module and are used to issue different commands to the NXT logo brick. The brick is the controller in the robot. It controls three powerful servos that pulls or pushes the trailer with the load. In this case, the three servos work in unison to generate maximum power. The brick also controls a sensor that collects information about the distance between the transmitter and the receiver.

The VI is designed to work as a RESTful web service, which can be invoked through the web. As a web service, it runs the code and then collects the current position of the robot through the sensors and creates a XML data with the available information and returns it. The robotic module has the following components:

\section{A. Control Movement of the Robot}

The robotic control unit (RCU) being applicationspecific, needs to be designed separately for each experiment. The robots contain one or more servos that are controlled by a LEGO NXT Brick, which in turn is controlled from the local RF server. The RCU has also some sensor for the collection of data about its current position after a request is completed. The RCU is controlled by invoking web services on the RF server, which sends corresponding signal to the NXT Brick. These units can perform complex movements such as, moving a device under test (DUT) in horizontal direction up to a certain distance.

\section{B. Safety and error correction}

Because a large number of inexperienced users (mostly undergraduate students) are likely to access the instrumentation, it is important to create as secure a network environment as far as possible. Specific issues considered include; accidental harm to the instruments, the instrument computer system, the software from the instrument vendor ('vendor software'), and the security of the data collected. Several strategies are adopted to achieve the goals include; a scheduling system which controls authentication and authorization via username, password, and reservation time. The instrument computer and vendor software are isolated from the remote user. As an example, towards safety of the instruments, in the antenna gain experiment considered here, the receiver antenna on the robot trailer need to be moved forward and/or backward within $100 \mathrm{~cm}$ (from the transmitter) to measure the noise and signal strength. If the user requests a movement beyond $100 \mathrm{~cm}$, then the robot control will be erroneous and even robot position may get disoriented. Hence, the LabVIEW control program has to be designed to ensure that the robot moves only up to $100 \mathrm{~cm}$. Also the robot with the receiver is expected to move with a movement accuracy of $1 \mathrm{~cm}$. To maintain the accuracy, additional sensors may be used to gather position information, the distance actually travelled and the current actual position of the receiver. The brick also need to control a sensor that collects information about the distance between the transmitter and the receiver.

\section{Data Acquisition}

After a request is processed, the current position or the status of the receiver unit must be relayed back to the user client. The user needs to know what changes has occurred to the setup since the last request was made. For this purpose, different sensors are connected to the RCU module to collect data for e.g., using ultrasonic sensor to measure distance traversed.

\section{CASE STUDY}

The NETLab system is being used for teaching RF concepts at the Indian Institute of Technology, Kharagpur. Below we present a case study on a specific experiment 'Maximum Operating Distance Measurement' of the Antenna gain experiment.

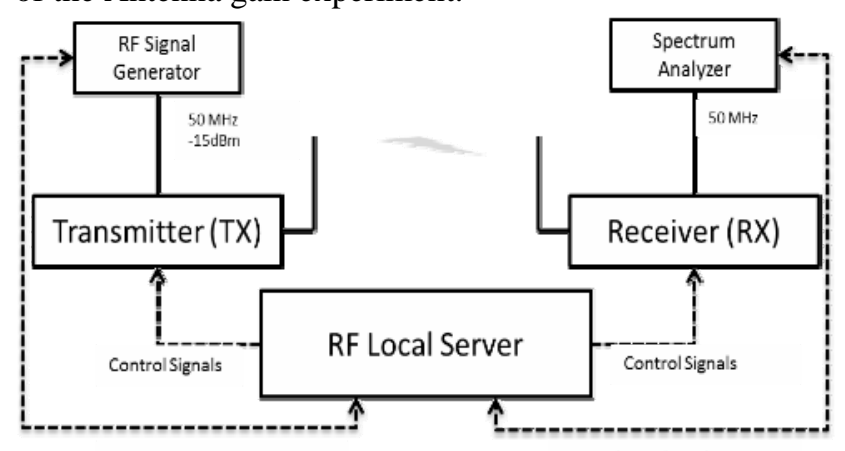

Control Signals and Data

Control Signals and Data

Figure 4. The experiment setup (flow).

\section{A. Problem description for the students}

For the experiment, the students are supposed to apply a $50 \mathrm{MHz}$ intermediate frequency (IF) signal in the transmitter (TX) unit. A spectral component of $50 \mathrm{MHz}$ should be observed appearing on the spectrum analyzer display. If the IF signal is turned off, only noise signal will appear on the spectrum analyzer's display in the vicinity of $50 \mathrm{MHz}$. Students need to measure the average noise power shown on the spectrum analyzer and finally calculate measure the signal-to-noise ratio (SNR) at the IF output of the receiver (RX) Unit. The aim is to 
PAPER

INTERNET-BASED ROBOT ASSISTED RF AND WIRELESS LABORATORY FOR ENGINEERING EDUCATION

determine the maximum distance between the TX and RX units to keep the SNR of at least 2 (see figures 3 and 4). The measurements need be done with power amplifier turned 'ON'. The students have to prepare a laboratory report on the results.

\section{B. Controlling the equipment}

The ME1000 main transmitter is turned 'on' or 'off' by LabVIEW module. The user can also turn 'on' or 'off' various other switches on the transmitter and receiver modules. The RF 'output' is obtained from AgilentN9310A. The RF measurement parameters are frequency and amplitude. A LabVIEW Web service is used to operate the instrument AgilentN9310A. On the receiver side AgilentN9320B RF spectrum analyzer is used. It is also controlled by LabVIEW Web services (see Figure 5).

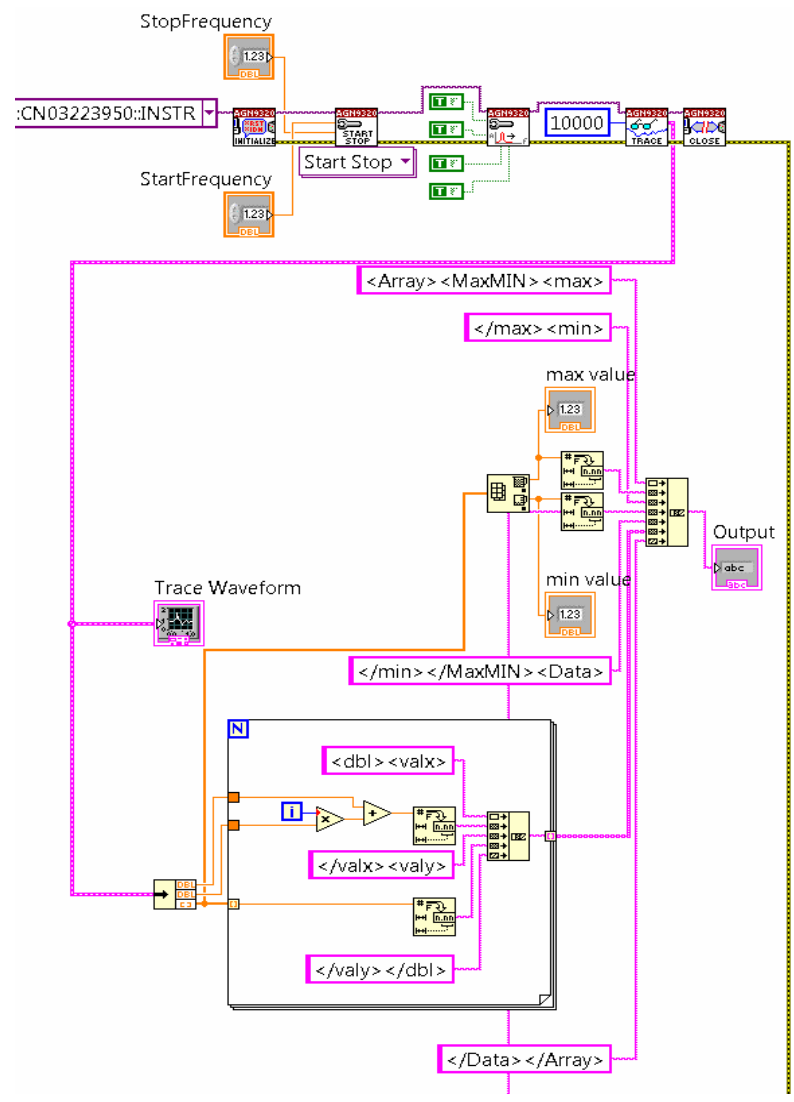

Figure 5. The VI file (partly) for data acquisition RF spectrum analyzer

\section{Controlling the Robot}

The experiment requires that the distance between the transmitter and receiver be changed to obtain maximum signal and to find the correct distance. To do this, the LEGO robot is designed as a carrier that moves the receiver (RX) mounted on top of it, along a table while the transmitter unit (TX) is fixed on the table. The NXT is also controlled by LabVIEW web services (see Figure $6)$. The user can control the movements from the user client (see Figure 2). The robot is attached with a sensor that records the distance between the transmitter and the receiver and sends back the information to the user client.
The safety is ensured by checking the current distance of the receiver unit from the transmitter.

\section{The User Interface}

The users log in to the system and open the user interface. Then the user can control the movement of the robot and see the current distance and save the current data for further analysis. The RF signal generator and RF spectrum analyzer settings can be altered. The student can turn 'on' or 'off' the IF amplifier settings and get the corresponding data sets from the interface. To aid teachers' online evaluation of student performance, provision has been made for keeping track of status at various stages of the experiment for each student and the evaluation is done. The process of evaluation is done in multiple steps as follows: Preliminary Quiz is conducted before the learner can access the actual experiment page. After the experiment has been performed successfully, the learner needs to save and plot graph etc., analyze data, interpret the results, and extract parameters, if applicable. The laboratory report should contain the detail on the experiment done as well as the data obtained and results and discussions. Once the laboratory report is submitted online by a student, it is accessible only to the instructor who may check it manually and grade the student. The student is now required to appear in viva-voce examination, which is again an 'online' evaluation and consists of several True/False questions that should be performed within a specified time.

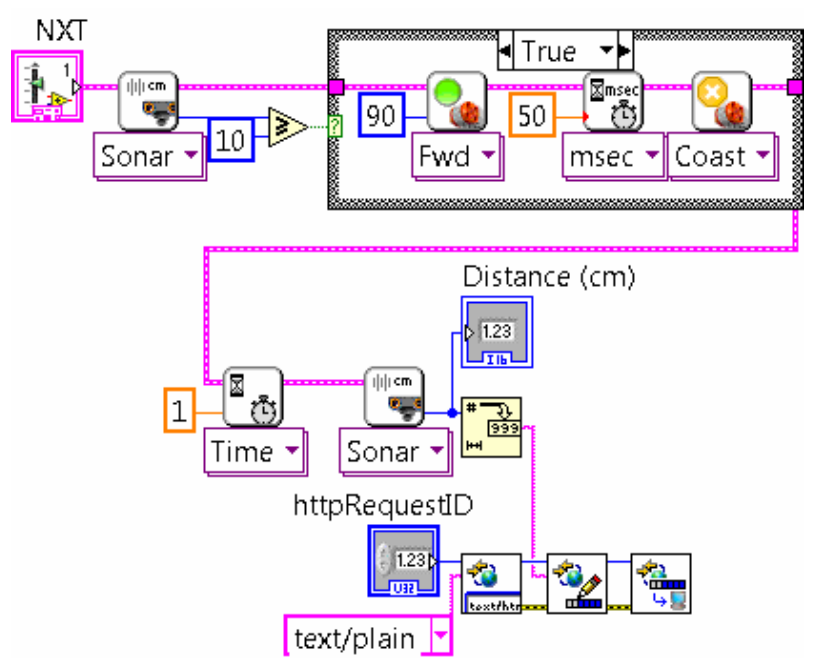

Figure 6. The VI file for NXT LEGO.

\section{DISCUSSIONS}

NETLab, an online Laboratory Management System (LaMS) in which several laboratory modules are included have been reported [4]. The application specific experiment 'Maximum Operating Distance Measurement' of the RF and wireless laboratory has been integrated with the NETLab. Our study indicates a growing interest of students in using sophisticated RF measurement equipments. This study is conducted as part of a ministry of human resources development (MHRD, New Delhi), project which aims at establishing the RF laboratory environment by enabling access to high technology remotely controlled robot-assisted radio communications 
equipment through the Internet. Currently, the project is in the pilot phase and is planned to be opened to students in future. However, the current version of the laboratory runs throughout the semester as an "open laboratory" where students are permitted to work $24 \times 7$. The students were given the opportunity to make specific comments regarding the experiment/laboratory. In addition to the online survey conducted for the students, the Department also conducted its own survey from the faculty to identify any significant deficiencies in the laboratory and specify actions to be taken to correct the deficiencies.

\section{EVALUATION}

Followings are some of the feedback from the students and their opinion about RF and microwave laboratory as a learning tool. This study is based on a student population of about 40 for the period July-December 2011. All the students used the same website that has the functionality such as choice of experiment, experimental procedure, laboratory manual, pre-laboratory theory etc. Interactive session was run with teachers and students, as well as an inquiry/feedback, which formed the basis for the evaluation. Laboratory evaluation procedure consists of several criteria, such as, Very Poor (VP), Poor (P), Fair (F), Good (G) and Very Good (VG) as measures. Typical results showed a distribution (in percentage) like this: Very Poor (3\%), Poor (5.5\%), Fair (9.7\%), Good (64.8\%) and Very Good (17\%). Laboratory assessment tools were used for evaluating the effectiveness of the laboratory experiments in terms of pedagogical benefits, resource utilization and cost effectiveness on a regular basis. Analytical tools were provided for each experiment to help in performing various analyses on the data collected during experiment.

\section{CONCLUSION}

Robot-assisted remote-controlled RF and wireless laboratory is reported. The laboratory is being used to impart training on RF and wireless basic concepts, measurements, instruments, and experiments that do not require any physical interaction between the student and the laboratory environment. A framework for robotassisted LabVIEW-based remote RF and wireless laboratory, accessible via the Internet by means of a Web browser is described in detail. Sharing of experimental resources between different institutes worldwide can now be enabled and the students outside campus can gain access to real laboratory experimentation online. The developed RF and wireless laboratory may provide an alternative practice platform for engineers and technicians who otherwise would not have the chance to access the laboratory. Introduction of a new platform with RF experiments to the NETLab laboratory management system (LaMS) will provide RF measurements and analyses platform for undergraduate education. Proposed internet-based robot-assisted laboratory will encourage institutions/universities to share the resources.

\section{ACKNOWLEDGMENT}

We are thankful to Dr. C K Maiti of IIT Kharagpur for technical discussions.

\section{REFERENCES}

[1] K. C. Gupta, T. Itoh, and A. A. Oliner, "Microwave and RF education past, present, and future,” IEEE Trans. Microwave Theory Tech., vol. 50, no. 3, pp. 1006-1014, Mar. 2002. http://dx.doi.org/10.1109/22.989984

[2] F. A. Cassara, "Wireless Communications Laboratory", IEEE Trans. Education, Vol. 49, pp. 132-140, 2006. http://dx.doi.org/ 10.1109/TE.2005.863428

[3] Dream Catcher, ME1000 RF Circuit Design Courseware, Quick Start Guide, ver 2.10.

[4] A. Maiti, "NETLab: An Online Laboratory Management System”, iJOE, vol. 6, Iss. 2, pp. 31-36, 2010.

[5] W. Kang and H. Ku, "Development of Web-Based Remote Laboratory for Education and Research on RF Engineering”, ICWL 2011, LNCS 7048, pp. 283-288, 2011.

[6] C. S. Tzafestas, N. Palaiologou, and M. Alifragis "Virtual and remote robotic laboratory comparative experimental evaluation," IEEE Trans. on Education, vol. 49, no. 3, Aug. 2006 http://dx.doi.org/10.1109/TE.2006.879255

[7] V.J. Harward, J.A. del Alamo, S.R. Lerman, P.H. Bailey, J. Carpenter, K. DeLong, C. Felknor, J. Hardison, B. Harrison, I. Jabbour, P.D. Long, Mao Tingting L. Naamani, J. Northridge, M. Schulz,D. Talavera, C. Varadharajan, Shaomin Wang, K. Yehia, R. Zbib and D. Zych, "The iLab shared architecture: a web services infrastructure to build communities of internet accessible laboratories,” IEEE, vol 96, no. 6. pp.931-950, Jun. 2008.

[8] C. C. Aydin, G. Turkmen, E. Ozyurt, E. U. Aydin, N. E. Cagiltay, M. E. Ozbek, N. C. Alparslan and A. Kara, "Distance laboratory applications ERRL: A study on radio communication in electronic field," 11th International Conference on Optimization of Electrical and Electronic Equipment, 2008. http://dx.doi.org/10.1109/OPTIM.2008.4602515

[9] J. A. Asumadu, R. Tanner, J. Fitzmaurice, M. Kelly, H. Ogunleye, J. Belter, and S. C. Koh "A Web-Based Electrical and Electronics Remote Wiring and Measurement Laboratory (RwmLAB) Instrument,” IEEE Trans. on Instrumentation and measurement, vol. 54, no. 1, Feb. 2005 http://dx.doi.org/10.1109/TIM.2004. $\underline{834597}$

[10] N. E. Cagiltay, E. Aydin, R. Oktem, A. Kara, M. Alexandru, and B. Reiner, "Requirements for remote RF laboratory applications: An educators' perspective," IEEE Trans. on Education, vol. 52, no. 1, Feb. 2009, pp. 75-81. http://dx.doi.org/10.1109/TE.2008. $\underline{919806}$

[11] N. E. Cagiltay, E. Aydın , C. C. Aydin, and A. Kara, "Seven Principles of Instructional Content Design for a Remote Laboratory: A Case Study on ERRL”, IEEE Transactions on Education, 54, 320-327,2011. http://dx.doi.org/10.1109/TE.2010. $\underline{2058115}$

[12] A. Maiti, Automatic Evaluation of Student's Performance in Online Laboratories, iJOE, vol. 6, Iss. 3 pp. 44-49 , 2010.

[13] C. F. Simón, L. M. Balada, O. G. Bellmunt, N. L. Martínez, O. B. Puxan, and R. V. Robles. "A Remote Laboratory Platform for Electrical Drive Control Using Programmable Logic Controllers," IEEE Trans. on Education, vol. 52, no. 3, Aug. 2009

[14] J. Murphy, I. Grout, J. Walsh, T. O’hea. "Local and remote laboratory user experimentation access using digital programmable logic," iJOE International Journal on Online Engineering - www.i-joe.org 1447

\section{AUTHORS}

Ananda Maiti is with the School of Computing Science and Engineering, Vellore Institute of Technology, Vellore 632 014, India (e-mail: anandamaiti@live.com).

S. Mahata is with the Department of Electronics and Electrical Communication Engineering, Indian Institute of Technology, Kharagpur 721 302, India (e-mail: subhasismahata@yahoo.com).

Manuscript received 09 March 2012. Published as resubmitted by the authors 21 July 2012. 